Patrick Schöfer*, Kornelius Lente, Celine Wegner, Andreas Kogut, Rüdiger Rupp and Thilo B. Krüger

\title{
A neuromonitoring framework supporting the intraoperative placement of an epidural spinal cord electrode array for functional restoration in people with paraplegia
}

\begin{abstract}
Targeted stimulation of dorsal nerve roots of the lumbosacral spinal cord (SC) represents a promising way to improve lower extremity functions for people with paraplegia due to SC injury. Stimulation pulses are selectively applied at different segments of the SC by means of an implanted electrode array. For an optimal clinical outcome, correct positioning of the array in rostrocaudal and mediolateral direction of the $\mathrm{SC}$ is essential. We developed a neuromonitoring framework to support the neurosurgeon during the implantation procedure by automated stimulation and real-time analysis of evoked muscular responses. For the implementation we developed and combined a new software with several hardware and software modules for intraoperative neuromonitoring. The new software coordinates different interfaces and components including a neurostimulator, a multiplexer module and a biosignal amplifier. In addition, a graphical user interface (GUI) was implemented, allowing control of the system and display of analyzed data. With the framework, stimulation sequences in respect to stimulation amplitudes and selection of contacts of the electrode array can be predefined and executed in an automated manner. Synchronously the evoked electromyographic responses of the target muscles can be recorded, analyzed and displayed together with the stimulation setup for visual evaluation. The framework serves as a fundamental basis for the integration of a clinical knowledge-based algorithm and the realization of a future neuromonitoring system for this purpose.
\end{abstract}

Keywords: Rehabilitation, Intraoperative Neuromonitoring,

\footnotetext{
*Corresponding author: Patrick Schöfer: University of Applied Sciences, Residenzstraße 8, 91522 Ansbach, Germany, e-mail: p.schoefer@gmx.de

Kornelius Lente, Thilo B. Krüger: inomed Medizintechnik $\mathrm{GmbH}$, Emmendingen, Germany

Celine Wegner: iNCU GmbH, Emmendingen, Germany Andreas Kogut, Rüdiger Rupp: Section Experimental Neurorehabilitation, Spinal Cord Injury Center, Heidelberg University Hospital, Heidelberg, Germany
}

Paraplegia, Targeted Stimulation, Electromyography

https://doi.org/10.1515/cdbme-2021-2017

\section{Introduction}

The motor control of human locomotion is a highly complex task involving different parts of the central nervous system in a hierarchical manner [1]. While the intention to perform a movement is generated in the brain, this intention is transmitted through the corticospinal tract of the SC to a spinal neuronal network, called spinal pattern generator (CPG), located in the lumbosacral SC. The spinal circuitry of the CPG is contributing to the generation of basic muscle activation patterns for unconscious walking. The CPG output is modulated by afferent input from muscle and joint afferents [2]. In case of an injury of the SC above the lumbosacral region, the efferent commands from the brain do not or only partially activate the CPG. As a result, the execution of movements is severely limited resulting in paralysis of the lower limbs.

Epidural electrical stimulation (EES) represents a promising approach for increasing the activity level of the CPG neural network by stimulation of dorsal SC nerve roots. Combined with task-oriented locomotion training, it might lead to a better recovery of voluntary muscle functions resulting in better ambulation [3], [4].

The central prerequisite for effective EES is to apply the pulses at the relevant segments of the SC. Within the European research project CONFIRM, we are developing a neuromonitoring system supporting the neurosurgeon in the intraoperative placement of an electrode array on the SC.

The fundamental principle of this system is the targeted stimulation by means of specific contacts of the electrode array and simultaneously monitoring of the evoked muscle activities in the lower extremities. By analyzing the recorded EMG signals, the selective activation of individual muscles and activation patterns of muscle groups is monitored and 
based on this, conclusions can be made regarding the need for repositioning of the electrode array.

\section{Methods}

\subsection{System setup}

The automated neuromonitoring framework is separated into two central tasks: Stimulation by means of selected contacts of the electrode array and recording of the evoked electromyographic responses with electrodes in up to 16 muscles of the lower extremities. The framework is implemented by coordinating an electrical stimulator, a biosignal amplifier, a multiplexer module and a neuromonitoring software with a new developed control software (inoInspect) (see Figure 1).

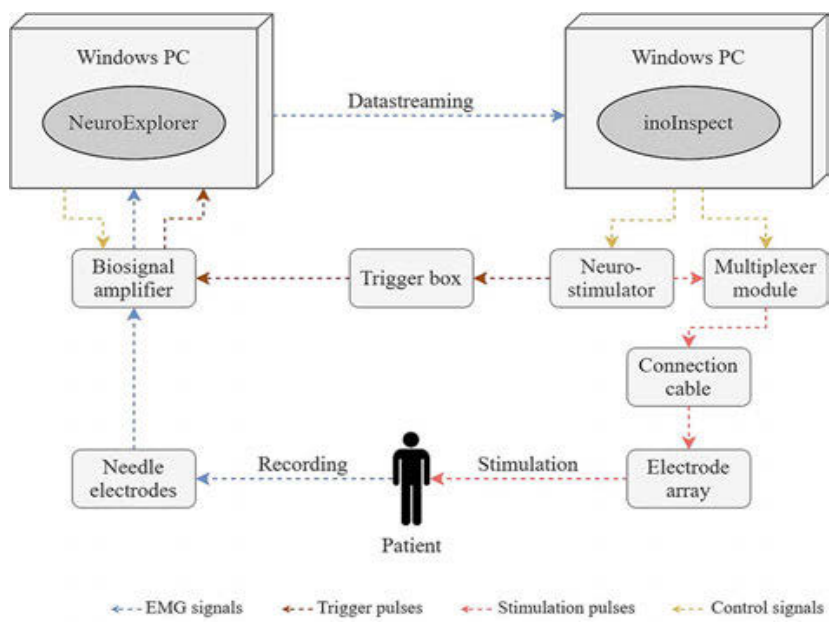

Figure 1: Setup and data flow of the automated neuromonitoring framework for targeted EES and electromyographic recording.

Via the GUI of the new software, parameters and electrode contacts of stimulation sequences can be defined. Afterwards the software coordinates the necessary interactions of the electrical stimulator with the multiplexer module (ISIS Neurostimulator, ISIS Switchmatrix, inomed Medizintechnik $\mathrm{GmbH}$ ) for the execution of the defined stimulation sequence. The neurostimulator contains twelve channels for high-current stimulation and one for direct nerve stimulation (DNS). The anode and cathode of the DNS are connected to the input of the 64-channel multiplexer module enabling software-controlled switching between different anodecathode combinations. Consequently, the generated pulses are transferred to the selected contacts of the electrode array and applied to different parts of the SC.

Triggered by the stimulation, electromyograms (EMG) of the muscles of the lower extremity are recorded with up to 16 needle electrodes (ISIS Headbox $16 \mathrm{Ch}$. DIFF, inomed Medizintechnik $\mathrm{GmbH}$ ) and displayed by the neuromonitoring software (NeuroExplorer, inomed Medizintechnik GmbH).

Additionally, the triggered EMG signals are directly streamed to inoInspect and displayed there along with the initiating stimulation setup.

\subsection{Software framework}

The new application-specific software inoInspect is written in Python (version 3.8) using an object-oriented development approach. Its architecture is based on a layer model consisting of three layers: presentation, logic and data. Within these layers, the software provides various functionalities along with related interfaces.

By implementing the GUI as the presentation layer of the software, an exchange of information in both directions is established. This enables the user to operate the system and its associated functionalities and allows the software to present the data generated during the positioning procedure. The GUI is developed with the standard GUI framework Tkinter.

The underlying logic layer processes user input and on this basis communicates with the two hardware modules for targeted stimulation execution. Moreover, in this layer the recorded signals are analyzed and a data flow between the data layer and the GUI is established.

The streamed signals from the NeuroExplorer are received in the data layer of the software and prepared in terms of their data structure for further processing. Moreover, an interface for interaction with the file system is provided here, which enables application-specific data to be imported and exported.

\subsubsection{Stimulation logic}

The stimulation is performed by means of adaptable stimulation patterns, which are based on different electrode configurations. Via a graphical representation of the electrode array, the user can create individual configurations and merge them into one stimulation pattern. An electrode contact can be defined as anode or cathode by clicking on it (see Figure 2). 


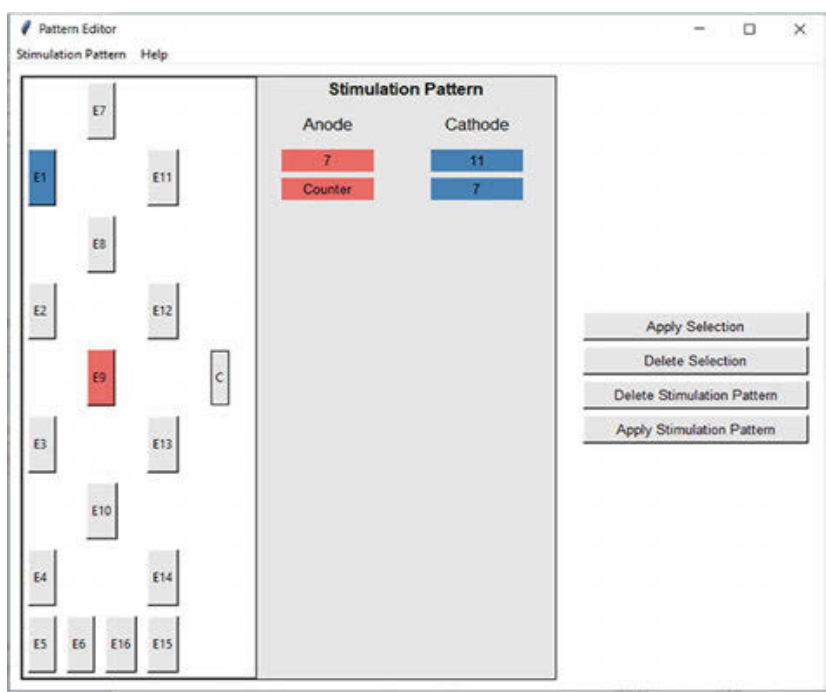

Figure 2: Screen of the Pattern Editor of the software. Two electrode configurations [anode $=7$, cathode $=11$ ] and [anode $=$ counter electrode, cathode $=7$ ] were selected in the example and merged to a stimulation pattern. The configuration [anode $=9$, cathode $=1$ ] has already been selected but not yet transferred to the pattern.

Each electrode configuration is associated with several stimulation intensities. They are specified by three parameters: start value, end value, and step size of the increasing current intensity. Based on this, the software calculates a ramp of stimulation intensities to fulfil the input parameters.

By activating the start button in the GUI, the parameters for generating a stimulation pulse are transferred from the software to the neurostimulator using a Dynamic-link library (DLL). The communication between the software and the multiplexer module enabling switching to the required electrode configuration takes place via DLL as well.

The software realizes a completely automated execution of a stimulation pattern. This means that once the upper limit of the stimulation ramp for one configuration has been reached, the software switches the electrode configuration by the multiplexer module and starts the next stimulation ramp of the pattern.

\subsubsection{Signal processing}

The recorded EMG signals are transferred to the new software using a server-client-architecture. The inoInspect software (client) requests the recorded triggered signals from the relevant measurement window of the NeuroExplorer (server). In response, after each triggered recording, it receives a stream of data blocks of the measuring channels. Since data streaming runs concurrently to the stimulation logic, an additional thread is created to parallelize the stimulation and the receipt of the signals.

Upon receipt, the data is sorted into a two-dimensional array structure and the amplitude of each signal is analyzed in respect to two thresholds previously defined by the user. Based on the result of the analysis, the signals are displayed in a particular colour on the GUI. The plot colour is set to green when both thresholds are exceeded, yellow when only the lower threshold is exceeded, and red when both are not exceeded. The signals are updated with each stimulation pulse.

For a direct comparison between one current intensity and the next within one electrode configuration, the latest signals are always shown in colour and the previous signals in light grey. To display earlier signals, a review function has been implemented. This has been programmed as an eventbased method, meaning that the user can click on the desired stimulation step in an overview field and all recorded signals of this electrode configuration are displayed. The signal corresponding to the stimulation parameters chosen is coloured in respect to the threshold analysis and the rest of the signals in light grey.

Storage of measurement data is performed in the background without any active request from the user necessary. The software creates a directory containing the entered information, the parameters, the executed electrode configurations, and the recorded signals in a structured way. In addition, a file is created, containing all the information for importing this measurement series.

\section{Results}

With the novel neuromonitoring framework the basic functionalities needed for supporting the intraoperative placement of an epidural stimulation electrode array by neuromonitoring methods are implemented. The system is able to switch between different contacts of the electrode array and an additional counter electrode. This allows bipolar stimulation, i.e., between two contacts of the array as well as monopolar stimulation, i.e., between one contact of the array and the counter electrode. Serval selected anode-cathode combinations can be merged to a stimulation pattern, which can be executed in an automatic, semi-automatic and manual way. Besides manual input of the stimulation patterns, it is also possible to import predefined patterns. In one electrode configuration, the system can automatically increase the 
A neuromonitoring framework supporting the intraoperative placement of an epidural spinal cord electrode array for functional restoration in people with paraplegia

current intensity stepwise according to the entered parameters.

The implemented GUI consists of a main window (see Figure 3) and three additional windows for entering the required data. These include a Pattern Editor for creating the stimulation pattern, a Parameter Editor for specifying the stimulation ramp and thresholds for signal analysis, and an Annotation Editor for entering additional information during the implantation procedure.

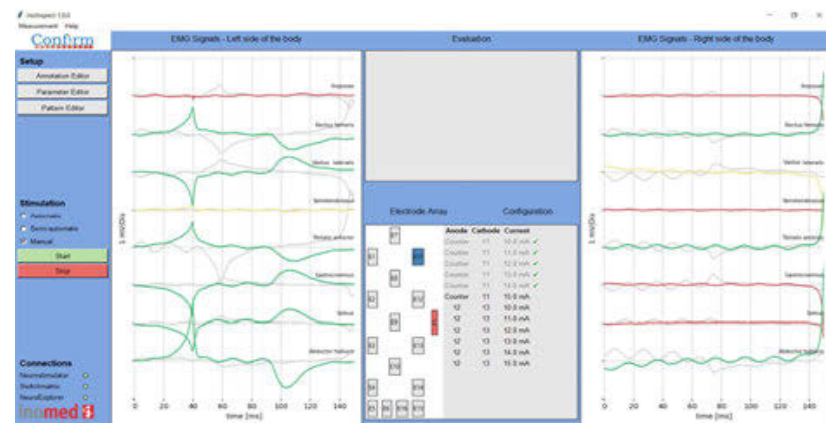

Figure 3: Main window of the GUI of the inolnspect software. Left: Control panel for opening the editors and control of the stimulation. Middle: Representation of the electrode array and overview of the stimulation steps. To its left and right, the signals are displayed according to the side of the body where they were recorded.

By classifying the muscle responses and displaying them in a corresponding colour code, the neurosurgeon or neurophysiologist quickly obtains an initial statement about the effect of the stimulation. As the signals are sorted vertically from proximal to distal muscles and horizontally into left and right, they can be directly compared with each other and consequently provide information about the correct position of the electrode array. The implemented logic of the review function enables switching between individual stimulation steps within the current measurement series as well as previous measurement series.

\section{Discussion}

In an initial approach of a CONFIRM-system, switching between contacts of the electrode array as well as generating a ramp of stimulation intensities had to be done in a manual way. The automated framework reduces the time required and improves the error-free and intuitive control of the system, the presentation of results and the documentation of relevant data.

As a next step, the new software will be tested in a clinical scenario without active control of hardware modules, but as a platform for visualisation and structured storage of application-specific data. This data will then be used for training and evaluation of an algorithm that is currently under development. In the future the system will be extended with this clinical knowledge-based algorithm to provide concrete guidance to the neurosurgeon about the directions of repositioning of the electrode array on the SC.

\section{Author Statement}

Research funding: This work was funded as part of the project CONFIRM by Eurostars (Grand number: 01QE1913B).

Conflict of interest: Kornelius Lente and Thilo B. Krueger are full-time employees of inomed Medizintechnik $\mathrm{GmbH}$. Celine Wegner is a full-time employee of iNCU GmbH.

Informed consent: Informed consent has been obtained from all individuals included in this study.

Ethical approval: The conducted research is not related to either human or animal use.

\section{Acknowledgment}

We thank the people involved in the CONFIRM project of the company Onward, the research institute École Polytechnique Fédérale de Lausanne and the company inomed Medizintechnik GmbH.

\section{References}

[1] O. Kiehn, 'Decoding the organization of spinal circuits that control locomotion', Nat. Rev. Neurosci., vol. 17, no. 4, pp. 224-238, Apr. 2016, doi: 10.1038/nrn.2016.9.

[2] E. Formento et al., 'Electrical spinal cord stimulation must preserve proprioception to enable locomotion in humans with spinal cord injury', Nat. Neurosci., vol. 21, no. 12, pp. 17281741, Dec. 2018, doi: 10.1038/s41593-018-0262-6.

[3] C. A. Angeli et al., 'Recovery of Over-Ground Walking after Chronic Motor Complete Spinal Cord Injury', N. Engl. J. Med., vol. 379, no. 13, pp. 1244-1250, Sep. 2018, doi: 10.1056/NEJMoa1803588.

[4] F. B. Wagner et al., 'Targeted neurotechnology restores walking in humans with spinal cord injury', Nature, vol. 563, no. 7729 , pp. $65-71$, Nov. 2018, doi: 10.1038/s41586-0180649-2. 